\title{
A Uniform Method of Mechanical Disturbance Torque Measurement and Reduction for the Seeker Gimbal in the Assembly Process
}

\author{
Naihui Yu and Jianzhong Shang \\ College of Mechatronics and Automation, National University of Defense Technology, Changsha, China \\ Correspondence should be addressed to Jianzhong Shang; jz_shang_nudt@163.com
}

Received 6 August 2017; Revised 29 September 2017; Accepted 9 November 2017; Published 4 December 2017

Academic Editor: Xuejun Xie

Copyright (C) 2017 Naihui Yu and Jianzhong Shang. This is an open access article distributed under the Creative Commons Attribution License, which permits unrestricted use, distribution, and reproduction in any medium, provided the original work is properly cited.

\begin{abstract}
In the manufacturing process of seekers, the reduction of disturbance torques (DTs) is a critical but time-consuming work. The innovation of the paper is to present a uniform method to measure and reduce mechanical DTs during gimbal's assembly process. Firstly, the relationships between assembly parameters and DTs are established and analyzed by theoretical model. And then, a measuring system is established to measure the driven torque of the gimbal's torque motor. With the goal of stabilizing and minimizing the driven torque, all assembly parameters relating to DTs could be adjusted. Through the proof of a lot of experiments, this proposed method could reduce the bias and fluctuation of these mechanical DTs. This method could also be used for the mechanical DTs reduction of most similar productions and improve the quality and efficiency during their system assembly process.
\end{abstract}

\section{Introduction}

The seeker is one most important subsystems of a missile, which is used to track the target and to provide the inertial stabilization to the detector's pointing vector [1]. It is generally composed of a detector, a controller for acquisition and tracking, and a multiaxis orthogonal gimbal system. In the task of long-range precision strike or target positioning, the stability of seeker's line-of-sight (LOS) is crucial to hit accuracy and target location accuracy of a missile. As a highly integrated and high precision optical-mechatronic system, there are many factors which might degrade the stabilization precision of LOS, such as disturbance torques, gyro noise and drift, structural resonance frequency, and control bandwidth $[2,3]$. Among these factors, DTs would cause nonlinear phenomenon, such as delay or distortion, of the whole system, so they are the key factors which would decide the dynamic performance of a seeker. Existence of the disturbance torque may corrupt the seeker tracking and severely degrade the guidance performance [4]. According to the summary of Masten MK, common gimbal disturbances are listed below [5].
Coulomb friction within the electromechanical assemblies is typically the dominant disturbance. Friction arises from surface interactions between and within the rotating bearings of the gimbals, environmental and electromagnetic interference seals, and brush contact in brush-type motors.

Spring torques are due to flexure, compression, or stretching of electrical cables between the payload and the host vehicle produce disturbances. In addition, some payloads require coolant lines or electrical or mechanical connections to the host vehicle.

Imbalance produces LOS jitter when the payload center of gravity is not centered on an axis of rotation for the gimbals. Linear vibration, acting through the lever arm of the center of gravity offset, thus produces torque disturbances.

Structural flexure occurs when a payload or gimbal assembly bends or deforms due to external disturbances or vibration. Jitter from structural bending is typically measured by the gyro, and control action is taken to reduce the effect. However, structural flexure can also occur in parts of the payload/gimbal assembly not measured by the gyro.

Vehicle motion (kinematic) coupling occurs when host vehicle maneuvers (pitch, yaw, and roll) couple into gimbal 
mechanisms. The gimbal orientation, relative to the host vehicle, clearly must change during such maneuvers, and the necessary change can be accounted by transforming the vehicle maneuvers into equivalent torque disturbances for the gimbal mechanisms.

Intergimbal coupling occurs when cross-products of inertia produce disturbances in one gimbal due to motion in another gimbal. Another interaxis coupling is gyroscopic torques, where the product of rates in two axes produces disturbances in a third axis.

Internal disturbances, including noise in the control loop components such as actuators, electronics, and gyros, can also be represented by an equivalent torque disturbance. Noise is usually defined as unpredictable disturbances.

Environmental disturbances are exhibited as both direct and indirect interactions. If a gimbal and payload are exposed to the vehicle's aerodynamic wind stream, direct buffeting occurs. Indirect disturbances include changes in temperature and ice buildup on the gimbal. Temperature variations can introduce changes in other disturbances such as friction or intergimbal coupling. Ice buildup can change gimbal balance so that vibration coupling is further aggravated.

Although these DTs have seriously negative impacts on the system, they cannot be thoroughly eliminated because of the intrinsic nature of a mechanical system. And the residual DTs might be exponentially magnified due to the high- $g$ maneuver, severe vibration, and vigorous temperature impact of a missile's operating environment. Therefore, all DTs must be attenuated to minimum. As the seeker is an integrated mechanical-electrical production, there are generally two methods to attenuate the impact of DTs: (1) from the control system, increase the stabilizing loop bandwidth to improve the disturbance rejection ability [6] or utilize advanced control methods to compensate DTs $[4,7]$; (2) from the mechanical system, improve machining precision of parts and assembly precision of the product to minimize the source of these DTs. Though there are plenty of control methods for rejection or compensation of DTs, excellent mechanical performance is a very fundamental requirement for a highperformance seeker. And if these DTs are too large or their nonlinearity is too overt, the control compensation methods could not work. Improving mechanical properties of a seeker should be the first strategy employed to correct stabilization problems.

The system assembly process (SAP) or integration process is a key stage to determine most DTs in the whole manufacturing process of a seeker. For example, axial preload of bearings would affect the amplitude values and fluctuations of frictional torque (FT); bending directions and fixed points of cross-axis electrical cables would change the properties of cable spring torque (CST); static and dynamic balancing procedure would decide the magnitude of the unbalance torque (UT). All operations which are carried out in SAP are uniformly named as assembly parameters in this paper. However, there are very few methods or devices to measure the DTs and control these assembly parameters in the practical assembly process. The results of SAP could only be verified in the final performance tests, while all DTs are blended into an integrated DT. And we do not know which assembly parameter is the main cause of the total DT and how to adjust these parameters. This would result in a very time-consuming procedure if the first assembly attempt is not successful, which means reiterative parameter adjustments and performance tests are unavoidable until performance indexes of the final assembly are qualified.

At present, most measuring methods and devices for seeker assembly could be only used for a certain kind of DTs. For UT, Boynton et al. [8] developed an instrument that measures the unbalance of gimbaled devices with sensitivity as high as $0.1 \mathrm{~g}-\mathrm{cm}$. Yu and Zhao [1] presented a novel method to measure UTs in a two axes gimbaled seeker using a measuring instrument with three weight sensors, but this method was insufficient for the higher performances of a seeker owing to the limited sensor's own accuracy. Yang et al. [9] developed a method to measure static unbalance of gimbal axis using drive current, and a balanced simulator is used to verify the proposed approach by the load and repeatability tests and the results showed that the developed measuring system is able to achieve a high precision with a greater stability. For FT, direct-driven gimbal is the research object of this paper, so the FT mainly comes from bearings. There are many devices designed for the measurement of bearing's FT $[10,11]$. And most factors relating to bearing's FT, such as axial load, operating speed, and lubricant, temperature [12-14], have been experimented and studied by many researches. However, there is almost no device specifically aimed at the FT measurement for seeker gimbal or other inertial stabilization platforms. For CST, Wang et al. [15, 16] presented a dynamic thin rod model to analyze the CST. A force gauge ( $0.02 \mathrm{~N}$ precision) is used to obtain the CST of the cable harness. But the imbalance and friction effect cannot be eliminated in this method. All above researches and devices cannot manage all DTs during SAP of a gimbal. And they face the same problem that a separate DT cannot be accurately measured due to the coupling of all DTs during the actual SAP of a gimbal. Therefore, all DTs should be considered uniformly.

In this paper, a uniform method to deal with mechanical DTs during SAP of a gimbal is presented. In the section of dynamic model and disturbance torques of the seeker gimbal, the dynamic model of a gimbal considering the DTs is established based on the rotor dynamic theory. In the local coordinate system, the unbalance torque could be divided into three vertical components and each plays different roles on the total unbalance torque. A model is established in Matlab/Simulink to simulate these effects and provide instructions for the actual balance process. For the FT of direct-driven gimbal, the model of bearing's FT is established and its relevant assembly parameters are analyzed. In the section of driven-torque measuring system and disturbance torque reduction, a driven-torque measuring system is constructed to measure the armature current of the torque motor that could be converted to driven torque by multiplying the electric torque coefficient of the motor. With the goal of stabilizing and minimizing the driven torque, all assembly parameters relating to DTs could be adjusted and some experimental results are presented to validate the performance of this measuring system. Based on this measuring 


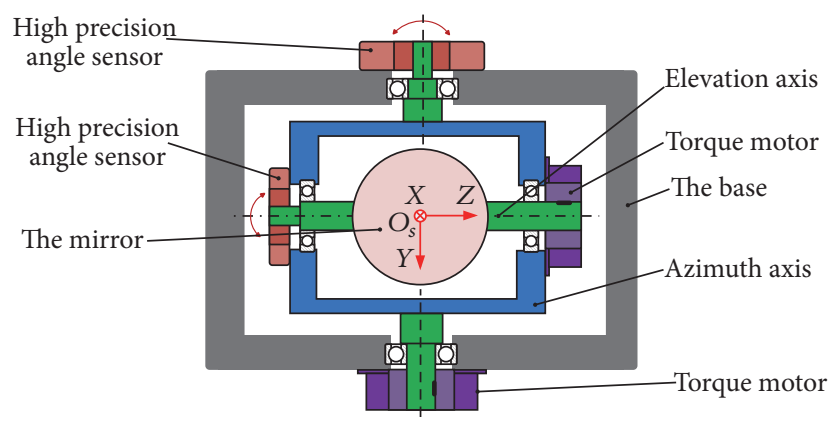

Figure 1: The conceptual graph of a seeker gimbal.

system, a uniform mechanical DTs reduction method during the SAP of a gimbal is presented. This method could reduce the mechanical DTs of the gimbaled-mirror system effectively and improve the efficiency of system assembly process.

\section{Dynamic Model and Disturbance Torques of the Seeker Gimbal}

As shown in Figure 1, it is the conceptual graph of a seeker gimbal. A two-axis seeker gimbal is composed of the azimuth and elevation axis, the payload (a detector or a mirror), and the base. Each axis contains a torque motor for driving and a high precision resolver for angular position feedback. If the payload is a detector, the gimbal is called direct pointing system. And if the payload is a mirror and the detector is attached on the base, it is indirect pointing system or gimbaled-mirror system, which orients a mirror in front of a detector so the reflected line-of-sight points in the desired direction [17]. It is obvious that the load mass of a gimbaled-mirror system is much lighter than that of direct pointing systems. This could significantly improve the resonance frequency and control bandwidth of servo mechanism. However, smaller load makes the seeker more sensitive to DTs and more difficult to stabilize the LOS of a seeker [18]. Therefore, much more attention should be paid on the DTs reduction during the fabrication of a gimbaledmirror seeker. In this paper, the gimbaled-mirror seeker is chosen as the research object.

To analyze the DTs of a seeker gimbal, its dynamic model should be established firstly. According to Newton's second law and the rotor dynamic theory, the basic motion equations for the azimuth and elevation axis could be directly obtained if the gimbals are considered as rigid bodies and mass unbalance is taken into account [19-22].

$$
\frac{d \vec{H}}{d t}=\vec{M}_{0}+\vec{\rho}_{c} \times\left(-m \vec{a}_{o}\right),
$$

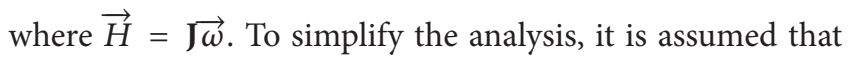
the gimbal rotation axes are aligned with the principal axes of inertia so that the inertia matrix is diagonal and $\mathbf{J}=$ $\operatorname{diag}\left(J_{x}, J_{y}, J_{z}\right) ; \vec{M}_{0}$ is the net torque vector imposed on the rotation axes; $\vec{\rho}_{c}$ is the moment-arm vector from center of gravity (CG) of rotation axes to geometric center $O_{s}$ of the seeker; $\vec{a}_{o}$ is the acceleration vector of geometric center $O_{s}$. So (1) could be expressed as

$$
\dot{\vec{\omega}}(t)+\vec{\omega}(t) \times \overrightarrow{J \omega}(t)=\vec{M}_{0}+\vec{\rho}_{c} \times\left(-m \vec{m}_{o}\right) .
$$

Expanding the above equation, the expression for dynamic model of rotation axis becomes

$$
\begin{aligned}
& {\left[\begin{array}{l}
M_{x} \\
M_{y} \\
M_{z}
\end{array}\right]} \\
& \quad=\left[\begin{array}{l}
J_{x} \dot{\omega}_{x}+\left(J_{z}-J_{y}\right) \omega_{y} \omega_{z}+m\left(\rho_{c y} a_{o z}-\rho_{c z} a_{o y}\right) \\
J_{y} \dot{\omega}_{y}+\left(J_{x}-J_{z}\right) \omega_{x} \omega_{z}+m\left(\rho_{c z} a_{o x}-\rho_{c x} a_{o z}\right) \\
J_{z} \dot{\omega}_{z}+\left(J_{y}-J_{x}\right) \omega_{x} \omega_{y}+m\left(\rho_{c x} a_{o y}-\rho_{c y} a_{o x}\right)
\end{array}\right] .
\end{aligned}
$$

If the revolving direction of a gimbal axis is $Z$ axis, its dynamic equation is

$$
M_{z}=J_{z} \dot{\omega}_{z}+\left(J_{y}-J_{x}\right) \omega_{x} \omega_{y}+m\left(\rho_{c x} a_{o y}-\rho_{c y} a_{o x}\right),
$$

where $M_{z}$ is a net torque including the driven torque, CST, and FT. So (4) could be expanded and rearranged as

$$
J_{z} \dot{\omega}_{z}=M_{z_{-} \text {motor }}-\left(M_{z_{-} \text {wire }}-M_{z_{-} f}-M_{z_{-} J}-M_{z_{-} u b}\right),
$$

where $M_{z \text { motor }}$ is the driven torque generated by the torque motor; $M_{z_{-} \text {wire }}$ is the nonlinear CST, and it is generally a function of angular position indicated as $\lambda_{z}$; namely, $M_{z_{-} \text {wire }}=f\left(\lambda_{z}\right) ; M_{z_{-} f}$ is the nonlinear FT, and it is a function of angular velocity and angular position [23]; namely, $M_{z_{-} f}=$ $f\left(\dot{\lambda}_{z}, \lambda_{z}\right) ; M_{z_{-} J}=\left(J_{y}-J_{x}\right) \omega_{x} \omega_{y}$ is the cross-coupling toque caused by product of inertia and in general, and it is more cost effective to incorporate in the initial design than the following assembly process [23]; $M_{z_{-u b}}=\rho_{c x} a_{o y}-\rho_{c y} a_{o x}$ is the UT. Except for $M_{z_{-} \text {motor }}$, all other torques are external imperfect DTs which would cause the jitter of LOS. There are four types of DTs and three of them could be reduced during the SAP.

It would be specially mentioned that each shaft of the gimbal is not a single movement. As the disturbance of the carrier (or missile) is inevitable, the servo mechanism has to make complex multiaxis motion in order to maintain the LOS stabilization in the space coordinate. The consequence is that they have an inherent base-motion coupling between the carrier and the LOS.

2.1. Analysis of UT. Due to the machining or assembling errors, the seekers are often not perfectly balanced and they generally have a CG offset relative to the gimbal rotation axes $[19,23]$. If the missile accelerates or sharply changes its directions, these accelerations have an adverse effect on imaging system performance. As the rotation range of the gimbaled-mirror seeker is usually small, the component of mass unbalance in $Y$ direction for elevation axis is usually considered as a more important component than that in $X$ direction, if the coordinate system is defined as Figure 1. And the same case would happen for the azimuth axis. However, 
it will be demonstrated that both components are critical for the total UT in the following section.

It is assumed that the linear acceleration of the missile's geometric center is $\vec{a}_{m}$ and its angular velocity and acceleration are $\vec{\omega}_{m}$ and $\dot{\vec{\omega}}_{m}$, respectively. The distance between the missile's geometric center and the seeker's geometric center $\mathrm{O}_{s}$ is $d_{s}$. According to the spatial geometric relationship and composition theorem of acceleration, the acceleration of $O_{s}$ is

$$
\vec{a}_{o}=\left[\begin{array}{c}
a_{o x} \\
a_{o y} \\
a_{o z}
\end{array}\right]=\left[\begin{array}{c}
-\omega_{m y}^{2} d_{s}-\omega_{m z}^{2} d_{s}+a_{m x} \\
\dot{\omega}_{m z} d_{s}+a_{m y} \\
\dot{\omega}_{m y} d_{s}+a_{m z}
\end{array}\right] .
$$

The projection of $\vec{a}_{o}$ in the direction of azimuth axis is

$$
\begin{aligned}
\vec{a}_{o A} & =\left[\begin{array}{l}
a_{o x A} \\
a_{o y A} \\
a_{o z A}
\end{array}\right]=R y_{A \leftarrow m} \cdot\left[\begin{array}{l}
a_{o x} \\
a_{o y} \\
a_{o z}
\end{array}\right] \\
& =\left[\begin{array}{ccc}
\cos \lambda_{y} & 0 & -\sin \lambda_{y} \\
0 & 1 & 0 \\
\sin \lambda_{y} & 0 & \cos \lambda_{y}
\end{array}\right]\left[\begin{array}{l}
a_{o x} \\
a_{o y} \\
a_{o z}
\end{array}\right] \\
& =\left[\begin{array}{c}
a_{o x} \cos \lambda_{y}-a_{o z} \sin \lambda_{y} \\
a_{o y} \\
a_{o x} \sin \lambda_{y}+a_{o z} \cos \lambda_{y}
\end{array}\right]
\end{aligned}
$$

where $R y_{A \leftarrow m}$ is the rotational transfer matrix from the base to azimuth axis and subscript $A$ represents azimuth axis. The projection of $\vec{a}_{o}$ in the direction of elevation axis is

$$
\begin{aligned}
\vec{a}_{o E} & =\left[\begin{array}{l}
a_{o x E} \\
a_{o y E} \\
a_{o z E}
\end{array}\right]=R z_{E \leftarrow A} \cdot\left[\begin{array}{l}
a_{o x A} \\
a_{o y A} \\
a_{o z A}
\end{array}\right] \\
& =\left[\begin{array}{ccc}
\cos \lambda_{z} & -\sin \lambda_{z} & 0 \\
-\sin \lambda_{z} & \cos \lambda_{z} & 0 \\
0 & 0 & 1
\end{array}\right]\left[\begin{array}{l}
a_{o x A} \\
a_{o y A} \\
a_{o z A}
\end{array}\right] \\
& =\left[\begin{array}{c}
a_{o x A} \cos \lambda_{z}+a_{o y A} \sin \lambda_{z} \\
-a_{o x A} \sin \lambda_{z}+a_{o y A} \cos \lambda_{z} \\
a_{o z A}
\end{array}\right],
\end{aligned}
$$

where $R z_{E \leftarrow A}$ is the rotational transfer matrix from the azimuth axis to elevation axis and subscript $E$ represents elevation axis.

Next, the UTs of the two-axis seeker will be analyzed. Firstly, we assume that the mass of elevation axis is $m_{E}$ and its $\mathrm{CG}$ is indicated as $\mathrm{O}_{c E}$. The moment-arm vector from $O_{c E}$ to $O_{s}$ is $\vec{\rho}_{c E}$; the mass of azimuth axis is $m_{A}$ and its CG is indicated as $O_{c A}$. The moment-arm vector from $O_{c A}$ to $O_{s}$ is $\vec{\rho}_{c A}$. So the UT of elevation axis in the revolving direction $(Z$ axis) could be expressed as

$$
M_{z E \_u b}=\left(\rho_{c x E} a_{o y E}-\rho_{c y E} a_{o x E}\right) m_{E} .
$$

And the UT in the other two unresolving directions ( $X$ and $Y)$ is

$$
\begin{aligned}
& M_{x E_{-} u b}=\left(\rho_{c y E} a_{o z E}-\rho_{c z E} a_{o y E}\right) m_{E}, \\
& M_{y E_{-} u b}=\left(\rho_{c z E} a_{o x E}-\rho_{c x E} a_{o z E}\right) m_{E} .
\end{aligned}
$$

Though $M_{x E_{\_} u b}$ and $M_{y E_{\_} u b}$ have no effect on the rotation of elevation axis, they will act on the rotation of azimuth axis as counter-moments. Assuming that $R z_{A \leftarrow E}$ is the rotational transfer matrix from elevation axis to the azimuth axis, the counter-moments caused by $\vec{M}_{E \_u b}$ can be calculated.

$$
\begin{aligned}
\vec{M}_{\text {back }} & =R z_{A \leftarrow E} \vec{M}_{E_{\_} u b} \\
& =\left[\begin{array}{ccc}
\cos \lambda_{z} & -\sin \lambda_{z} & 0 \\
\sin \lambda_{z} & \cos \lambda_{z} & 0 \\
0 & 0 & 1
\end{array}\right] \cdot\left[\begin{array}{c}
M_{x E \_u b} \\
M_{y E \_u b} \\
M_{z E_{\_} u b}
\end{array}\right] .
\end{aligned}
$$

Therefore, the counter-moment acting on the rotation direction ( $Y$ direction) of the azimuth axis is

$$
M_{y A \_b a c k}=M_{x E \_u b} \sin \lambda_{z}+M_{y E \_u b} \cos \lambda_{z} \text {. }
$$

The UT of azimuth axis, which is caused by its own mass unbalance, in the revolving direction ( $Y$ axis) could be expressed as

$$
M_{y A \_u b}=\left(\rho_{c z A} a_{o x A}-\rho_{c x A} a_{o z A}\right) m_{A} .
$$

So the total UT of azimuth axis is

$$
M_{o y_{-} t}=M_{y A \_u b}+M_{y A \_b a c k} .
$$

As can be seen from the above derived equations, the UT of a gimbal is not only affected by CG offset $\vec{\rho}_{c}$ but also by angular disturbance of missile $\vec{\omega}_{m}$ and $\dot{\vec{\omega}}_{m}$, linear acceleration $\vec{a}_{m}$, the rotation angle of frames $\lambda_{y}$ and $\lambda_{z}$, the distance $d_{s}$, and so on. A simulation model based on these equations is established using Matlab/Simulink. The simulation parameters are set according to a practical missile and its seeker gimbal, including $m_{E}=0.37 \mathrm{~kg}, m_{A}=0.47 \mathrm{~kg}$, $\vec{a}_{m}=\left[\begin{array}{lll}392 & 20 & 20\end{array}\right]^{T} \mathrm{~m} / \mathrm{s}^{2}$, and $d_{s}=4 \mathrm{~m}$. The angular disturbance of the missile is a sinusoidal motion and its amplitude and frequency are 1 degree and $3 \mathrm{~Hz}$. The work mode of the seeker is set as the search mode, so the rotation of gimbal's two axes is a sinusoidal motion within a range of -15 degree +10 degree, while its frequency is $1 \mathrm{~Hz}$. The simulation time is set as $30 \mathrm{~s}$.

If $\vec{\rho}_{c E}=\left[\begin{array}{lll}0.5 & 0.5 & 0\end{array}\right] \mathrm{mm}$, the UT of elevation axis (red) is shown in Figure 2. And if there is only its component, namely, $\rho_{c x E}=0.5 \mathrm{~mm}$ or $\rho_{c y E}=0.5 \mathrm{~mm}$, the UT is also calculated and shown in Figure 2. The statistical results of these UT, including mean value (MV) and standard deviation (STD), are shown in Table 1.

From Figure 2 and Table 1, we can find that $\rho_{c x E}$ is the main cause of UT fluctuations while its STD is much larger; $\rho_{c y E}$ is the main cause of UT amplitude while its MV is much 
TABLE 1: Statistical results of UT of elevation axis.

\begin{tabular}{lccc}
\hline & $\begin{array}{c}\text { Total unbalance } \\
\text { (red) }\end{array}$ & $\begin{array}{c}\text { Only } \rho_{c x E} \\
\text { (blue) }\end{array}$ & $\begin{array}{c}\text { Only } \rho_{c y E} \\
\text { (black) }\end{array}$ \\
\hline $\begin{array}{l}\text { MV } \\
(\mathrm{Nmm})\end{array}$ & -63.92 & 6.76 & -70.68 \\
$\begin{array}{l}\text { STD } \\
(\mathrm{Nmm})\end{array}$ & 11.92 & 10.91 & 1.54 \\
\hline
\end{tabular}

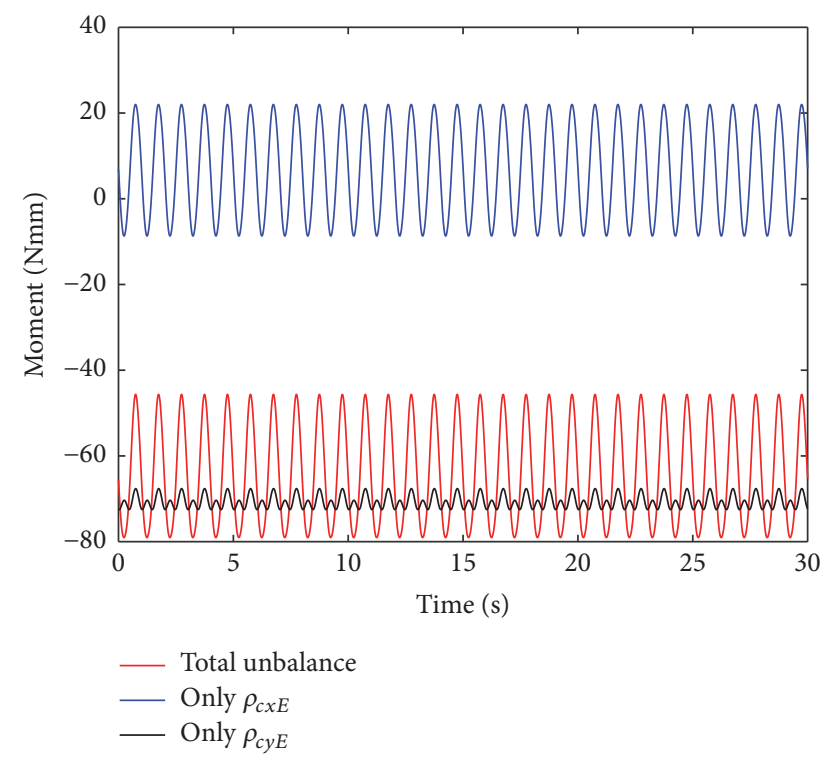

FIgURE 2: The UT of elevation axis.

larger. $\rho_{c x E}$ and $\rho_{c y E}$ play quite different roles on the total UT, but both should be attenuated as far as possible.

For azimuth axis, the mass unbalance of azimuth and elevation axis would both generate UT. So it is assumed that $\vec{\rho}_{c E}=\left[\begin{array}{lll}0.5 & 0.5 & 0.5\end{array}\right] \mathrm{mm}$ and $\vec{\rho}_{c A}=\left[\begin{array}{lll}0.5 & 0 & 0.5\end{array}\right] \mathrm{mm}$ as the total unbalance. Then the total UT is calculated, which is shown in Figure 3 (red). Besides, all components are simulated as an independent input. The MV and STD of undulant torque are also calculated and presented in Table 2.

As shown in Figure 3 and Table 2, $\rho_{c z E}$ of elevation axis and $\rho_{c z A}$ of azimuth axis are main causes of UT amplitude, while their MVs are much larger. $\rho_{c x E}$ of elevation axis and $\rho_{c x A}$ of azimuth axis are main causes of UT fluctuations. Besides, $\rho_{c y E}$ of elevation axis has little effect on azimuth axis, while its MV and STD are both very small.

From the simulation results, it could be found that the fluctuation of UT is very severe because of the high- $g$ maneuver and angular disturbance of a missile, although the mass unbalance is static and linear. And if the vibration of the missile is also considered, the fluctuation would become much more severe. Therefore, both mass unbalances in two vertical directions of rotating axis are crucial. In the SAP of a gimbal, $\rho_{c z E}$ of elevation axis and $\rho_{c z A}$ of azimuth axis could be balanced together in the static balance of azimuth axis, while $\rho_{c z E}$ has no effect on elevation axis.
2.2. Analysis of Nonlinear FT. Nonlinear FT is normally the major stabilization error source for various precision servo mechanisms [18]. The nonlinear behavior caused by friction has an adverse influence on the ultra-low velocity and high precision position control of servo mechanisms [24], which could lead to stick-slip and limit cycles during movement of inner and outer gimbals [25]. For direct-driven gimbals, the friction mainly comes from bearings. During the SAP, many assembly parameters, such as the pretightening force, assembly interference, lubricant, and running-in time, have important influences on the properties of the FT. What is special for a missile is that ambient temperature would be very different because of the broad geographical deployment and different seasons, and the environment temperature might change in a wide range of $-40^{\circ} \mathrm{C} \sim 60^{\circ} \mathrm{C}$ or much more severe. The effect of temperature on the FT should not be neglected [26].

In general, bearing's FT is derived from the elastic hysteresis of contact materials, lubricant viscosity, ball spin sliding friction, friction between rolling elements and cage, and so forth [27].

(i) Rolling Friction due to Elastic Hysteresis of Contact Materials. When a ball rolls on raceways, front and back of contact regions have asymmetric pressure distributions because of elastic hysteresis of contact materials. Resistance moment generated by front contact region is higher than driving torque generated by back contact region, so a rolling friction is produced. Based on Hertz contact theory, the friction torque of the bearing axis caused by one ball in the inner and outer raceway can be expressed, respectively, as [28]

$$
\begin{gathered}
M_{E I}=\frac{3 \varepsilon_{r} b_{I} Q_{n}}{16 R_{I}}\left(\frac{d_{m}}{2}-\frac{D_{w} \cos \alpha}{2}\right), \\
M_{E O}=\frac{3 \varepsilon_{r} b_{O} Q_{n}}{16 R_{O}}\left(\frac{d_{m}}{2}+\frac{D_{w} \cos \alpha}{2}\right),
\end{gathered}
$$

where subscripts $O$ and $I$ represent inner and outer rings, respectively; $Q_{n}=F_{a} / Z \sin \alpha$, if only axial preload $F_{a}$ is considered. Therefore, bearing friction torque caused by hysteresis is the sum of friction torques of all balls and is shown as

$$
M_{R}=\sum_{j=1}^{z} M_{E I, j}+\sum_{j=1}^{z} M_{E O, j}
$$

(ii) Sliding Friction due to Pivoting on Contact Ellipse. Spin motion of steel balls would happen in the ball bearing's running process and sliding friction is produced. Based on Hertz contact theory similarly, sliding friction torque on the elliptical contact is shown in the following equation [28].

$$
\begin{aligned}
& M_{S, j}^{\prime}=\frac{3 \mu Q_{n}}{2 \pi a b} \int_{-a}^{+a} \int_{-b\left[1-(x / a)^{2}\right]^{1 / 2}}^{+b\left[1-(x / a)^{2}\right]^{1 / 2}}\left(x^{2}+y^{2}\right)^{1 / 2} \\
& \times\left[1-\left(\frac{x}{a}\right)^{2}-\left(\frac{y}{b}\right)^{2}\right]^{1 / 2} d x d y=\frac{3}{8} \\
& \cdot \mu Q_{n} a L_{E} .
\end{aligned}
$$


TABLE 2: Statistical results of UT of azimuth axis.

\begin{tabular}{lcccccc}
\hline & Total unbalance & $\begin{array}{c}\text { Only } \rho_{c x E} \\
\text { (blue) }\end{array}$ & $\begin{array}{c}\text { Only } \rho_{c y E} \\
\text { (black) }\end{array}$ & $\begin{array}{c}\text { Only } \rho_{c z E} \\
\text { (green) }\end{array}$ & $\begin{array}{c}\text { Only } \rho_{c x A} \\
\text { (magenta) }\end{array}$ & $\begin{array}{c}\text { Only } \rho_{c z A} \\
\text { (cyan) }\end{array}$ \\
\hline MV (Nmm) & 163.31 & -0.59 & 1.68 & 71.75 & -0.67 & 91.14 \\
STD (Nmm) & 25.66 & 10.93 & 1.26 & 0.61 & 14.15 & 0.78 \\
\hline
\end{tabular}

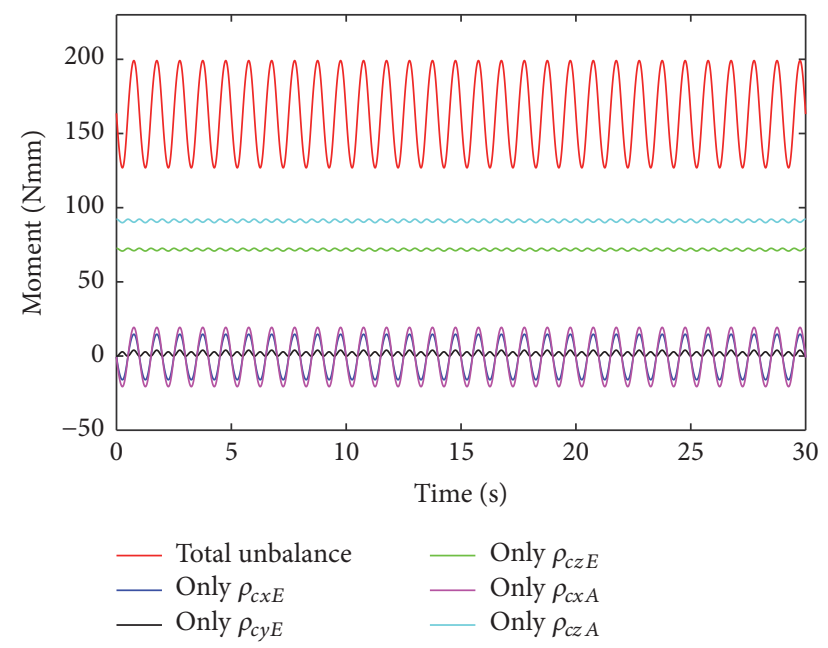

FIgURE 3: The UT of azimuth axis.

For the ball sliding in the inner or outer raceway is controlled by the maximum contact friction force, so $M_{S}^{\prime}$ is the maximum of $M_{S I}^{\prime}$ and $M_{S O}^{\prime}$. And the total sliding friction torque can be calculated in

$$
M_{S}^{\prime}=\sum_{j=1}^{Z} \max \left(M_{S I, j}^{\prime}, M_{S O, j}^{\prime}\right) .
$$

(iii) Friction Torque due to Lubricant Viscous. Palmgren (1959) has established experimental equations for friction torque caused by lubricant viscous, which is shown in

$$
M_{L}= \begin{cases}10^{-7} s_{0}\left(v_{0} n\right)^{2 / 3} d_{m}^{3}, & v_{0} n \geq 2000 \\ 160 \times 10^{-7} s_{0} d_{m}^{3}, & v_{0} n<2000 .\end{cases}
$$

If the grease is used, the decisive factor of $v_{0}$ is the viscosity of the base oil at operating temperature.

(iv) The Total Friction Torque and Ball Load. The total friction torque of bearing is the sum of all friction torque components and can be show in

$$
M=M_{R}+M_{S}+M_{L} .
$$

As can be seen from (15), (17), (19), and (20), there are many factors which would affect the bearing's FT, for example, geometric parameters, axial preload, lubricant viscous, and rotating speed.

During the SAP of a direct-driven gimbal, many assembly parameters have coupling influences on its FT. For example, the interference fit usually used for bearing assembly would compress the bearing's outer ring and meanwhile enlarge its inner ring, which inevitably results in the decrease of contact angle. According to (15), the contact angle of bearing would change the FT. And meanwhile the axial preload would also change the bearing's FT. With these two parameters enlarging, the FT would increase rapidly. As the interference amount is always difficult to be precisely measured, the control of axial preload becomes more important.

Though smaller preload means smaller FT, this assembly force must have enough magnitude to eliminate the bearing clearance, which is very crucial for rotating accuracy of gimbal axis. Besides, axial force is a very important factor for the bearing stiffness and also the resonant frequency of the total system [29]. Therefore, the requirement of axial preload presents a tradeoff on the FT, the structural stiffness, and rotating accuracy of the gimbal.

The above analysis only involves the first two components of bearing's FT and the third one (presented in (19)) has a connection with lubricant viscous. Through a series of experiments, Wikström and Höglund [12] have proved that the most important thing to start-up torque was base oil viscosity for grease-lubricated rolling element bearings at low temperatures. For most types of lubricant, their viscosity will exponentially rise with the temperature dropping. So the bearing's FT will also rise dramatically at a low temperature. To decrease the friction torque due to lubricant viscous, the type and the amount of lubricant should be tested before the SAP of a gimbal.

Another critical problem for FT is its fluctuations at different angular positions. Many factors would cause the friction fluctuations, so this problem is complex and difficult to establish its mathematical model. In the practical engineering application, running-in procedure is an important method to solve this problem in the assembly process. However, in the tradition assembly process, the runningin procedure depends on the manual operation and the running-in effect cannot be visible and controlled.

\section{Driven-Torque Measuring System and Disturbance Torque Reduction}

To control the assembly parameters relating to DTs during SAP of a gimbal, the measurability for all DTs is necessary. For DC torque motors which are usually used in the direct-driven seekers, its output torque is generally linear-proportional to its armature current. This provides an alternative method to conveniently measure all DTs. In a steady state, the balance equation of motor torque can be expressed as

$$
M_{\text {motor }}=M_{\text {out }}+M_{0}=K_{T}\left(I_{\text {out }}+I_{0}\right),
$$


where $K_{T}$ is the electric torque coefficient of the motor; $M_{\text {out }}$ and $I_{\text {out }}$ are the load torque of motor and its corresponding current, respectively; $M_{0}$ and $I_{0}$ are the no-load output torque and its corresponding current of motor, respectively, which are commonly provided by the motor manufacturers.

If the rotation axis is controlled to rotate uniformly, the driven torque of the motor is mainly used to overcome the DTs, which means

$$
M_{\text {motor }}=M_{\text {wire }}-M_{f}-M_{J}-M_{u b}
$$

According to (21), the armature current of torque motor must be measured accurately and electric torque coefficient should be calibrated carefully. According to (22), the key problems are how to accurately measure the driven torque and how to identify every DT from the measured driven torque.

A separate motor calibration device is set up for $K_{T}$ calibration, including a DC torque motor using in the gimbal, a servo driver for motor driving, a programmable multiaxes controller (PMAC) for motor control, a micro-range torque sensor for small torque measurement, and a Data Acquisition Card for torque value recording. The PMAC is used to drive the torque motor from static to starting and read its current when the motor just begins rotating. At the same time, the Data Acquisition Card was used to record the torque value from the torque sensor. Using these data, a calibrated value of $K_{T}$ could be got. Different weights were used to change the load of moment motor and we can obtain an accurate value of current-torque relation by taking the average of all measurement data. After the calibration of $K_{T}$, a driventorque measuring system (DTMS) is established. At each step of the SAP, we can measure the armature current of the motor and obtain the driven torque when the motor is controlled to rotate at a constant speed.

3.1. Measurement and Reduction of UT. It is assumed that there is a UT $M_{z E_{-} u b}$ on the elevation axis. In the actual rotating range, namely, $-15^{\circ}$ to $+10^{\circ}$, the change range of UT is $M_{z E_{\_} u b} \cos \left(-15^{\circ}\right)$ to $M_{z E \_u b} \cos \left(+10^{\circ}\right)$ or $M_{z E \_u b} \times 0.9659$ to $M_{z E\lrcorner u b} \times 0.9848$. The change is so small that it could be considered as a constant value.

Firstly, the DTMS is used to drive and control the motor to rotate at a constant speed, and meanwhile the driven torque is measured for the forward and reverse rotating directions. As shown in Figure 4, the dynamic torque of two directions (blue) has an offset from zero to the same direction and with nearly the same size. The MV of forward direction torque is $8.6172 \mathrm{Nmm}$, while the MV of reverse direction torque is $-2.7142 \mathrm{Nmm}$. So the torque offset value is $2.9515 \mathrm{Nmm}$. A ballast weighting $10 \mathrm{~g}$ is added to the rotation frame at the position of $30 \mathrm{~mm}$ biased from rotating axis, which means $3 \mathrm{Nmm}$ counterbalance torque is added. Then another similar measurement is carried out and its result is shown in Figure 4 (red). The MV of forward direction torque is $5.5961 \mathrm{Nmm}$ and the MV of reverse is $-5.5070 \mathrm{Nmm}$, which means the residual UT is very small. After that, we rotate the elevation axis for 90 degrees and repeat the above measuring steps to balance $\rho_{c x E}$.

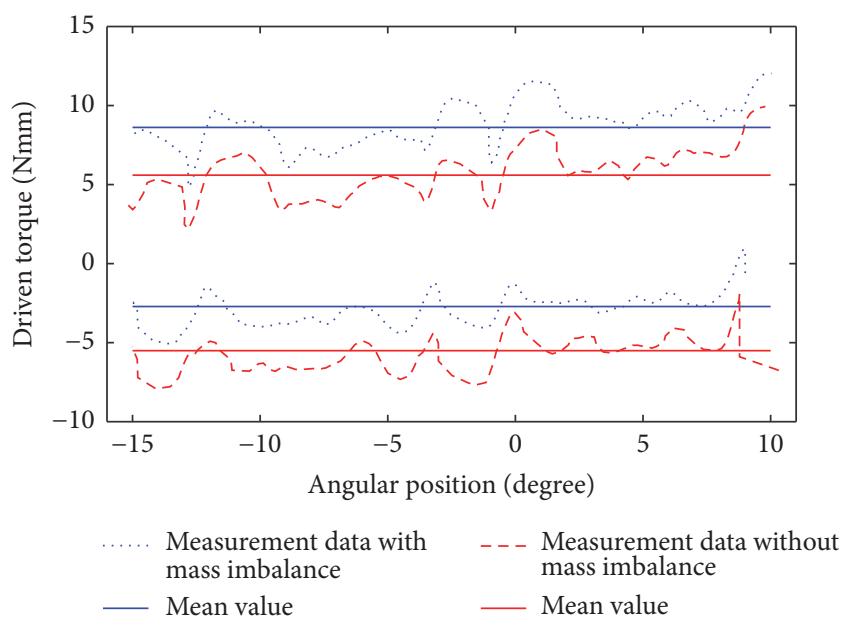

FIGURE 4: Measurement data and diminution of UT.

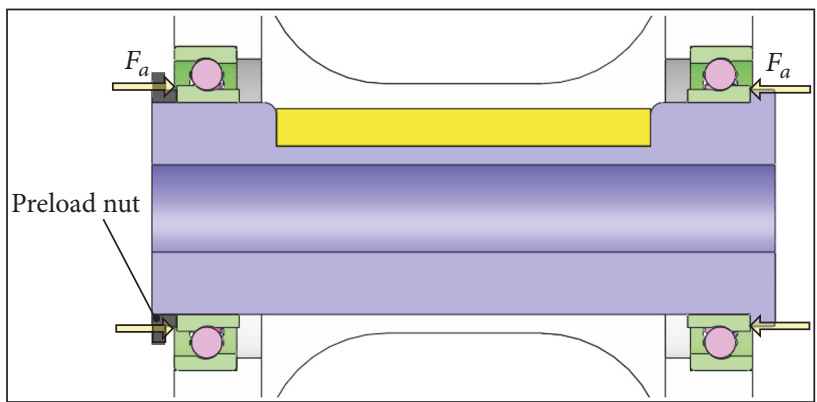

FIGURE 5: Adjustment method of axial preload.

For azimuth axis, using the same operations, the unbalance toque of two directions could be reduced.

Utilizing the DTMS to measure the driven torque and attenuate UT is a very convenient method. Though the counterbalance precision is not very high, our goal in this step is to reduce the offset of driven torque as far as possible. So the precision could be tolerated as the following diminution for other DTs will be carried out.

\subsection{Measurement and Reduction of FT and Its Fluctuations}

3.2.1. The Effect of Axial Preload on the FT. According to the analysis of nonlinear FT, the control of the bearings axial preload is very important for the performance of a gimbal. As shown in Figure 5, a preload nut is used to adjust the axial force $F_{a}$ and externally applied moments on the nut generate different axial preloads, which would change the FT of the bearings.

In the following assembly experiment, a digital screwdriver is used to apply and measure the pretightening moment on the preload nut and its measurement range is $\pm 2000 \mathrm{Nmm}$ and measurement precision is $1 \%$. The driven torque of the motor is measured by the DTMS. Nine different pretightening moments are, respectively, applied on the preload nut in this experiment, and its range and interval are $100 \mathrm{Nmm} \sim 500 \mathrm{Nmm}$ and $50 \mathrm{Nmm}$. The measurement 


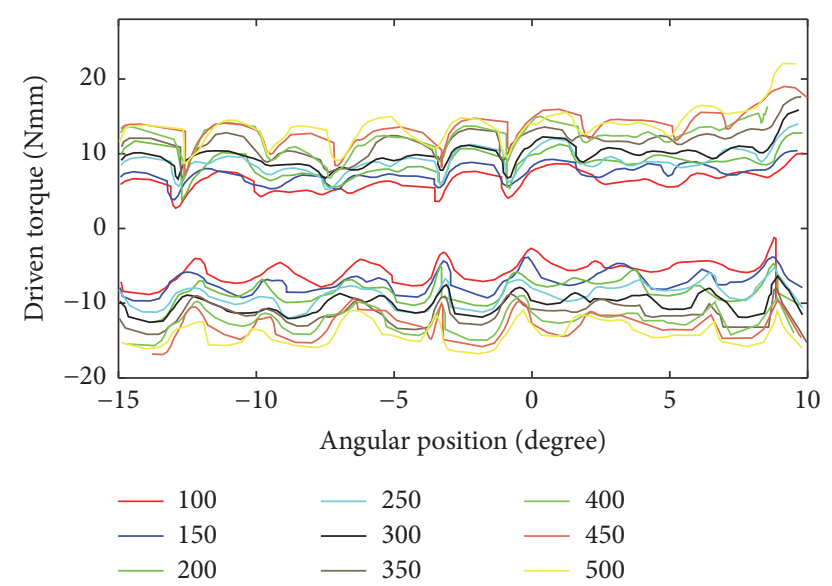

FIGURE 6: Measuring curves of driven torque versus different applied moments.

results of driven torque are shown in Figure 6. The values of driven torque increase obviously along with the increase of axial preloads. In these measuring curves, most peaks or valleys appear at the same angular positions; this means that the fluctuations of the FT are not stochastic and they are determined by certain surface qualities of bearing's raceways. The MVs and STDs of all measuring curves are calculated and shown in Figures 7(a) and 7(b), respectively.

As shown in Figure 7(a), the MVs of measuring curves almost linearly grow with the increase of applied moments. In order to better illustrate this relationship, the least square method is used to fit the parameters of a first-order function and its slope is $0.01981 \mathrm{Nmm} / \mathrm{Nmm}$, which means that the MVs of FT increase $1.981 \mathrm{Nmm}$ if the applied moments increase $100 \mathrm{Nmm}$. The STDs of measuring curves generally grow with the increase of applied moments but not linearly, which is shown in Figure 7(b). Therefore, the increase of applied moments results in more dramatic fluctuation of FT.

3.2.2. The Effect of Grease and Temperature on the FT. The properties of bearing's lubricant have a great influence on its FT, especially in a low temperature environment. In this paper, three types of grease named as \#1, \#2, and \#3 are chosen to lubricate the bearings of the gimbal and to conduct comparison experiments. Besides, the driven torque of a gimbal without any lubricant is also measured. To simulate the changing temperature, the gimbal is placed in a rapid temperature change test chamber. And the friction torque of its elevation axis is measured by DTMS at different temperatures whose range is $-40^{\circ} \mathrm{C}$ to $+60^{\circ} \mathrm{C}$. The MVs of measured driven torque at different temperatures and for different lubricants are presented in Figure 8.

From Figure 8, it could be found that driven torque of the gimbal without lubricant is lowest and it keeps almost stabile at all temperature points. Though the lubricant has an adverse effect on the FT at a low temperature, it is necessary for bearings because of its other functions, for example, wear reduction, impurity prevention, and heat dissipation. Among three selected greases, \#1 grease has the best performance and the increase of driven torque is slowest as the temperature dropping. For \#3 grease, the driven torque increases sharply and the motor could not be started when the temperature drops to $-30^{\circ} \mathrm{C}$. Based on DTMS, the grease suitable for a low temperature could be quickly selected. What is more essential, during the SAP of a gimbal, injecting quantity of grease could also be confirmed using this method, which means a lowest quantity should be used if other functions are meet.

3.2.3. Fluctuations of FT and the Running-In Procedure. The fluctuations of FT at different angular positions are a very detrimental problem for servo control and LOS stabilization of a seeker. During SAP of a gimbal, running-in procedure, which is just to make the shaft rotate repeatedly by artificial way, has many functions, such as to grind off some initial tiny defects of contact surfaces, to spread the lubricant more evenly in the bearings, and to release some concentrated stress caused by assembly force or machining process. And all these functions will help reduce the fluctuations of FT. In this paper, after assembly process of a gimbal is finished, DTMS is used to drive the motor and to conduct the running-in procedure automatically. During this procedure, the driven torque could be measured in real time and the performance of running-in could also be monitored. The measured results of a running-in procedure are shown in Figure 9.

As shown in Figure 9, the initial driven torque (blue) has very severe fluctuations at different angular positions, while its MV is $12.91 \mathrm{Nmm}$ and STD is $2.19 \mathrm{Nmm}$. After the automatic running-in procedure is carried out for 8 hours, the fluctuations significantly decrease, while its MV is $11.80 \mathrm{Nmm}$ and STD is $1.17 \mathrm{Nmm}$. So the running-in procedure could not only reduce the fluctuations but also decrease the amplitude of FT. However, the reducing effect is gradually weakening if more time is used. After operating for 24 hours, the measuring curve (red) is shown in Figure 9, while its MV and STD are $11.51 \mathrm{Nmm}$ and 0.93 , respectively. As the runningin procedure is a time-consuming process, the real time monitoring is very useful to improve efficiency during the SAP of a gimbal.

3.3. Measurement of Spring DT and Cables Fastness. As shown in Figure 10(a), to protect and house the cables, they always have to be combined and placed into a harness. As shown in Figure 10(c), in order to fix these cables harness, solid sols are generally needed to glue it in some fixed or fastness points. For a light load servo mechanism, the spring disturbance torque caused by cable harness has severe effects on the control system. By choosing different route, free length, and fastness position, cable routing can significantly determine the disturbance torque [16]. This CST is generally nonlinear and is very hard to establish its accurate mathematical model. This makes the reduction of this CST more difficult in the design stage and the adjustment in SAP is avoidable.

In this paper, the initial driven torque of a gimbal is measured using the DTMS when the cable harness is not fastened, and the result is shown in Figure 10(b). The parameters of a first-order function for the forward and reverse measuring curves are fitted with the least square 


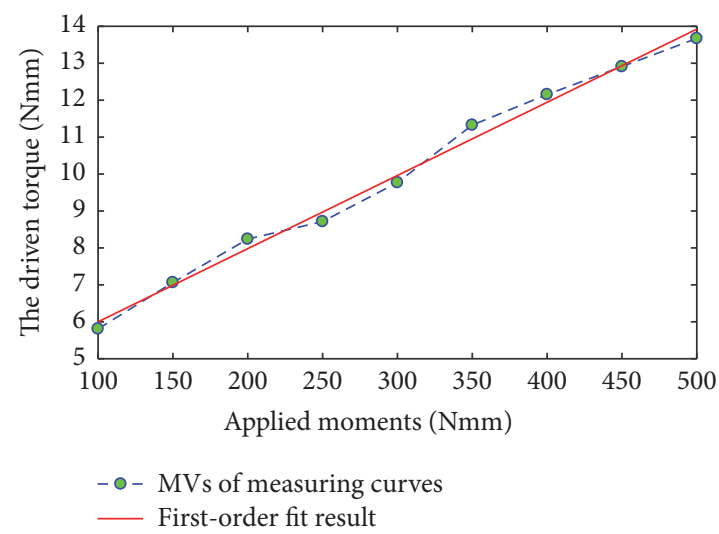

(a)

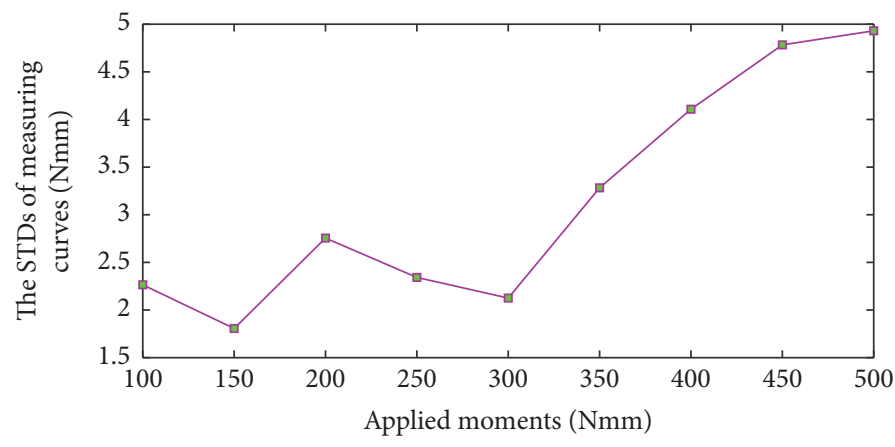

(b)

FIgURE 7: The MVs and STDs of measuring curves versus different applied moments. (a) The MVs. (b) The STDs.

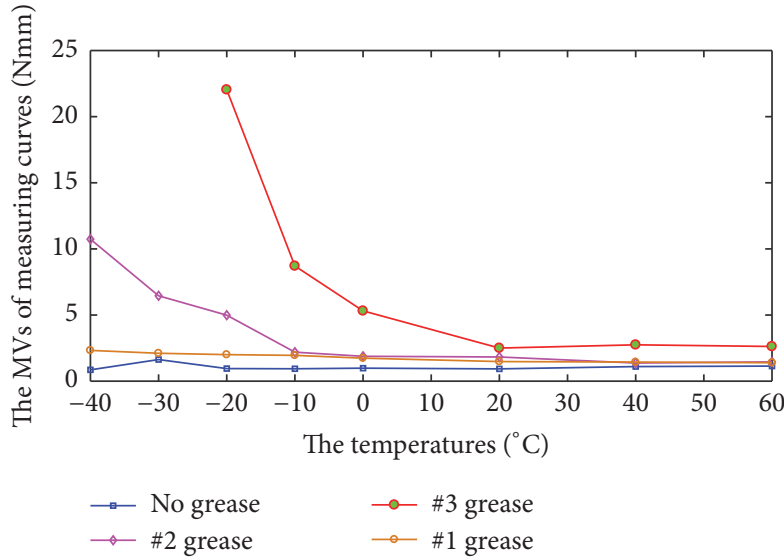

FIGURE 8: Driven torques of the gimbal at different temperatures.

method. The slopes of the fitted lines are 0.1510 and 0.0087 , respectively, which means the spring torque is very small if the cable harness is not fastened. When the cable harness is fixed by solid sols (Figures 10(c) and 10(d)), the slopes are 0.6174 and 0.4613 . It could be found that an improper cable routing and fastness method will result in a rotation of the measuring curves of driven torque. Though the rotation cannot be avoided, we could adjust the fastness points or cable route to decrease this DT.

3.4. A DTs Diminution Method during the SAP of a Gimbal. As we all know, during the SAP of a gimbal or other similar productions, assembly parameters relating to geometric accuracy could be controlled by various measuring equipment, for example, dial indicators, coordinate measuring machines, and laser tracking meters. However, there are very few measuring methods applicable to mechanical DTs, and assembly parameters relating to DTs are usually adjusted by workers' experience during assembly process. This could cause serious randomness of production quality or uncontrolled quality. So

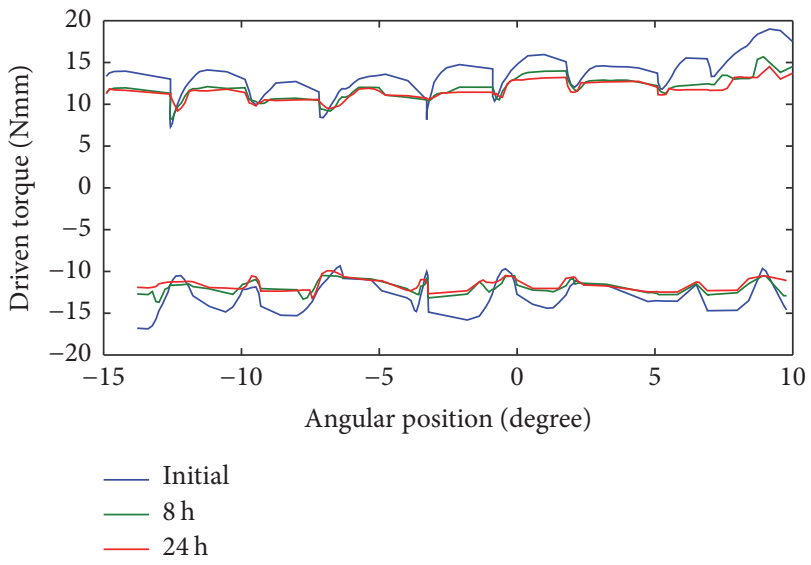

FIGURE 9: The measured results of a running-in procedure.

a uniform measuring method and criteria used to manage the mechanical DTs during SAP of a gimbal are urgently needed. According to the analysis and experiments in this paper, the DTMS could measure most DTs during the SAP of a gimbal. And the goal of this method is to make the driven current or driven torque steady as far as possible.

As shown in Figure 11, the whole SAP of a gimbal could be divided into mechanical system assembly, running-in procedure, mass balance procedure, cable fastness procedure, and so on. In the specific assembly steps, running-in procedure is to minimize the fluctuation of driven torque, mass balance procedure is to minimize the offset value of driven torque, and cable harness fastness procedure is to minimize the scope of driven torque. All these assembly steps could utilize the DTMS to real-timely monitor the driven torque and adjust the relevant assembly parameters. Therefore, this method is to distribute the reduction of total gimbal DT to each assembly step and control the assembly parameter during SAP.

It should be specially mentioned that the adjustment of bearings' axial preload has an opposite effect on the driven torque and the radial run-out of rotating axis. During this 


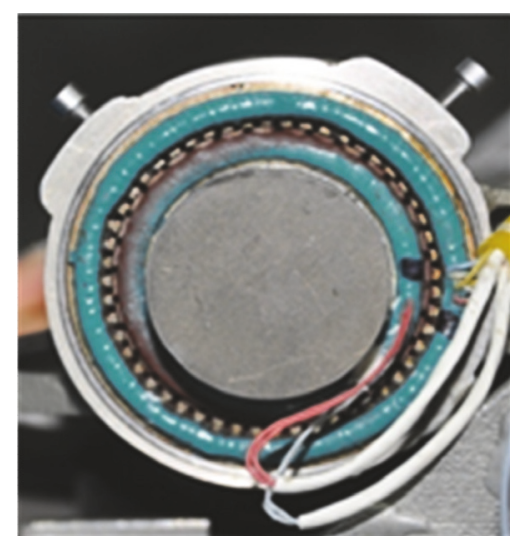

)

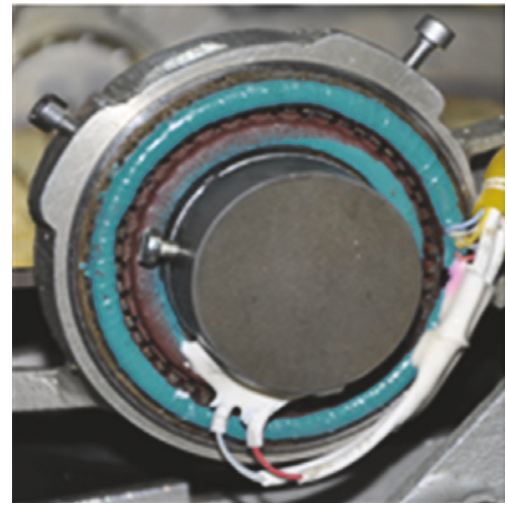

(c)

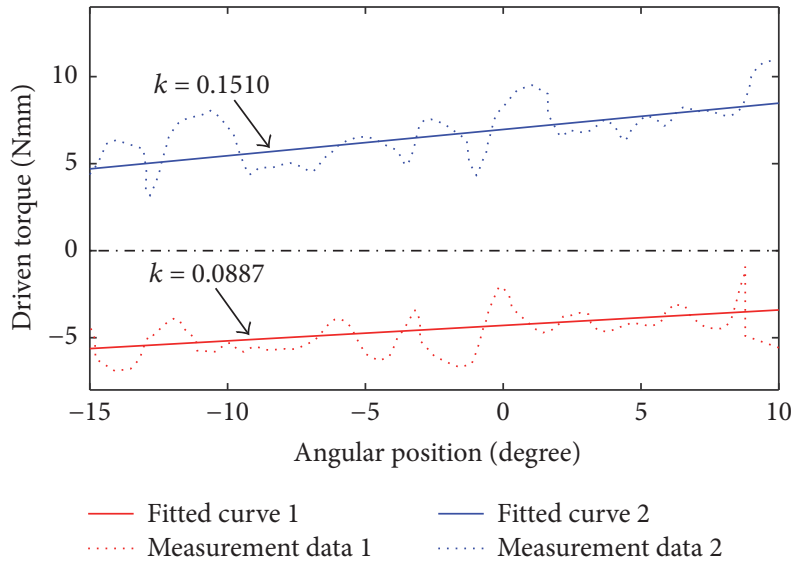

(b)

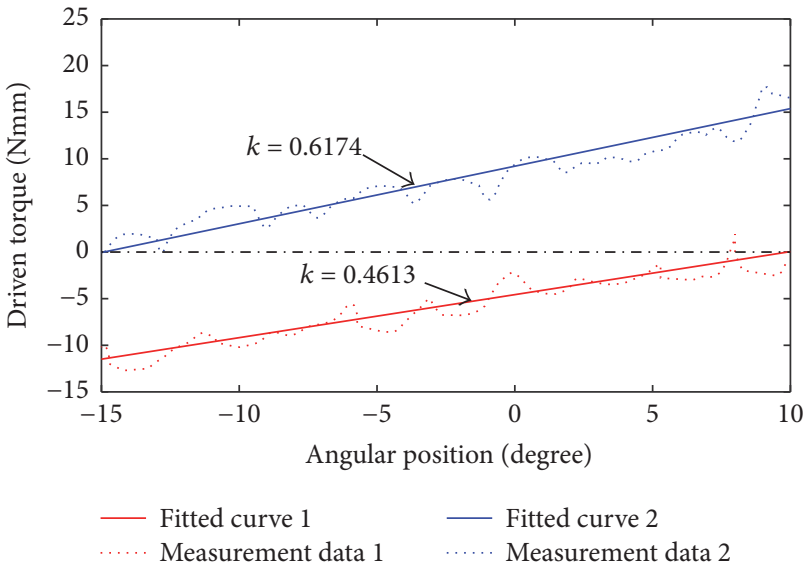

(d)

FIGURE 10: Cable harness fastness and measurement data of spring torque. (a) Free state of cable harness. (b) The measurement data of free state. (c) Fastness state of cable harness. (d) The measurement data of fastness state.

assembly step, an autocollimator should be used to measure the radial run-out when the axial preload is adjusted.

\section{Conclusion}

Based on the measurement of the motor's driven-torque, a uniform method for mechanical DTs reduction is presented in this paper. To confirm the influence factors of the UT and FT, theoretical models are established and analyzed. For UT, both mass unbalances in two vertical directions of rotating axis are crucial and play different roles on the total UT. For the FT, the interference fit and the axial preload have coupling effects on the value of FT. Because the interference amount is usually uncertain, the control of axial preload is more important.
In order to control the assembly parameters relating to mechanical DTs during SAP of a gimbal, a driven-torque measuring system for the motors is established. In a small rotation range, the UT would cause an offset of the driventorque. Using the DTMS, an exact weight of the counterbalance ballast could be calculated. For the FT, the axial preload adjustment, the grease selection, and the running-in procedure could be monitored. The spring torque caused by cable harness would result in an obvious slope of the driventorque. Therefore, the fastness position and cable routing adjustment should be optimized to minimize this slope.

All above work could be used to reduce the mechanical DTs of the seeker gimbal. It is proved by experiment that this method could greatly improve assembly efficiency and quality. 


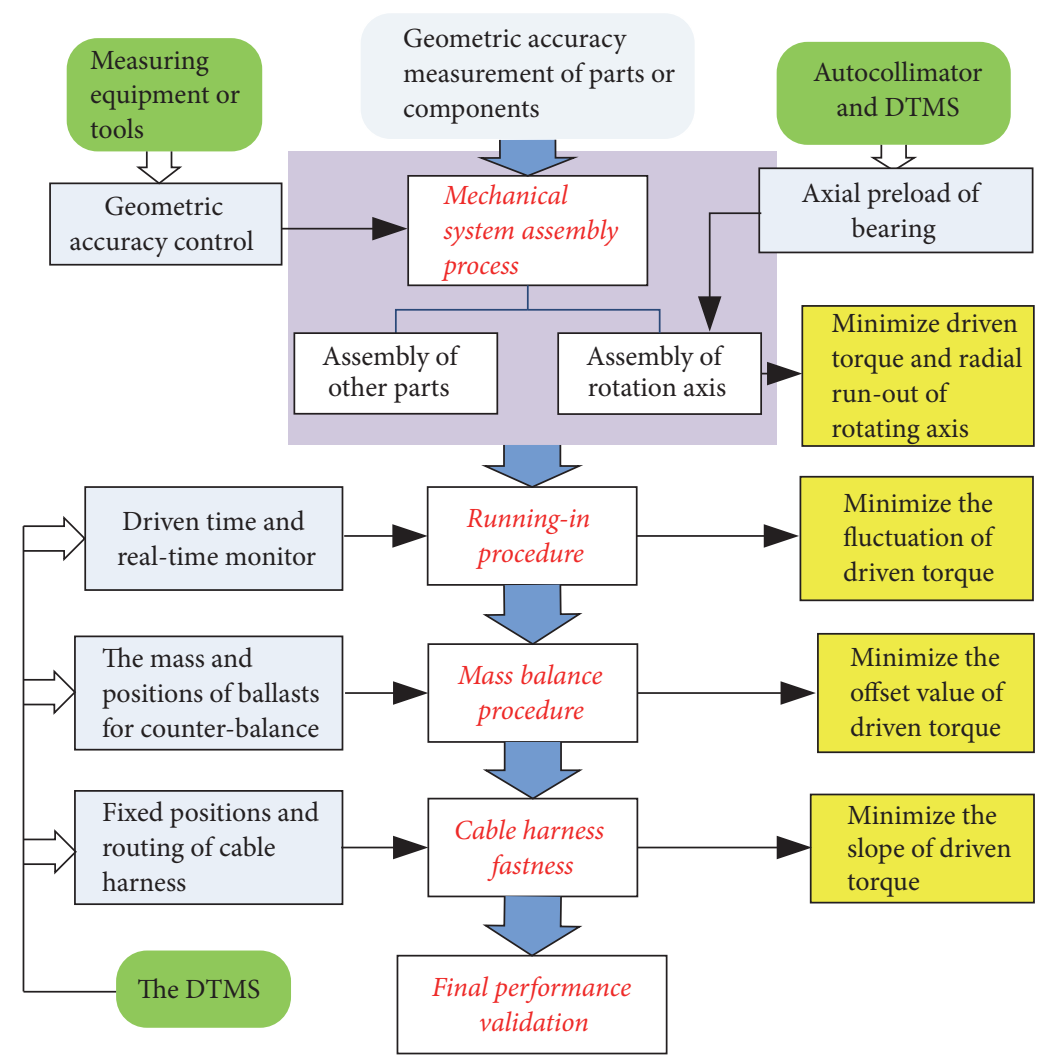

FIGURE 11: A disturbance reduction method during the SAP of a gimbal based on DTMS.

\section{Glossary of Defined Acronyms and Variables}

Defined Acronyms

CG: Center of gravity

CST: Cable spring torque

DT: Disturbance torque

DTMS: Driven-torque measuring system

FT: $\quad$ Frictional torque

LOS: Line-of-sight

PMAC: Programmable multiaxes controller

SAP: System assembly process

UT: Unbalance torque.

\section{Variables for Gimbal Dynamic Model}

$\vec{H}$ : The angular momentum

$J$ : The inertia matrix

$\vec{\omega}$ : The angular velocity vector

$\dot{\vec{\omega}}$ : The angular acceleration vector

$\vec{M}$ : The torque vector

$\vec{\rho}$ : The moment-arm vector

$m$ : The mass

$\vec{a}$ : The acceleration vector.

Variables for Bearing Friction Model

$Q_{n}$ : Ball-raceway normal load

$b$ : The half width of the ball-race contact ellipse
$R:$ The radius of the curved Hertzian contact

$\varepsilon_{r}$ : The energy loss percentage because of elastic hysteresis, generally, $\varepsilon_{r} \leq 1 \%$ for metal

$d$ : The groove diameter of raceway

$D_{w}$ : The ball diameter

$d_{m}$ : The mean bearing diameter

$\alpha$ : The contact angle of the ball with the raceway

$F_{a}$ : The axial preload

$a$ : The half length of the ball-race contact ellipse

$\mu$ : The coefficient of sliding friction

$L_{E}$ : The elliptic integral of second kind

$s_{0}$ : A dimensionless parameter relating to bearing type and lubrication method

$v_{0}$ : The kinematic viscosity of lubricant at operating temperature

$n$ : The operating speed

$Z$ : The ball number.

\section{Conflicts of Interest}

The authors declare that there are no conflicts of interest.

\section{Acknowledgments}

This work was supported by the National Natural Science Foundation of China (Grant no. 51675527).

\section{References}

[1] S. Yu and Y.-Z. Zhao, "Simulation study on a friction compensation method for the inertial platform based on the disturbance 
observer," Proceedings of the Institution of Mechanical Engineers, Part G: Journal of Aerospace Engineering, vol. 222, no. 3, pp. 341346, 2008.

[2] J. M. Hilkert, "Inertially stabilized platform technology: concepts and principles," IEEE Control Systems Magazine, vol. 28, no. 1, pp. 26-46, 2008.

[3] G. Downey and L. Stockum, "Electro-optical tracking systems considerations," in Proceedings of the SPIE conference on Acquisition, Tracking, and Pointing III, vol. 1111, pp. 70-84, Orlando, FL,USA, September 1989.

[4] C.-L. Lin and Y.-H. Hsiao, "Adaptive feedforward control for disturbance torque rejection in seeker stabilizing loop," IEEE Transactions on Control Systems Technology, vol. 9, no. 1, pp. 108-121, 2001.

[5] M. K. Masten, "Inertially stabilized platforms for optical imaging systems: tracking dynamic targets with mobile sensors," IEEE Control Systems Magazine, vol. 28, no. 1, pp. 47-64, 2008.

[6] J. M. Hilkert and B. Pautler, "A reduced-order disturbance observer applied to inertially stabilized Line-of-Sight control," in Proceedings of the 25th SPIE Acquisition, Tracking, Pointing, and Laser Systems Technologies XXV, vol. 8052, pp. 1-12, Orlando, FL, USA, 2011.

[7] B. Li, D. Hullender, and M. DiRenzo, "Nonlinear induced disturbance rejection in inertial stabilization systems," IEEE Transactions on Control Systems Technology, vol. 6, no. 3, pp. 421-427, 1998.

[8] R. Boynton, K. Wiener, P. Kennedy et al., "Static balancing a device with two or more degrees of freedom," in Proceedings of the 62nd Annual Conference of Society of Allied Weight Engineers, New Haven, Conn, USA, article 3320, May 2003.

[9] H. Yang, Y. Zhao, M. Li, and F. Wu, "The static unbalance analysis and its measurement system for gimbals axes of an inertial stabilization platform," Metrology and Measurement Systems, vol. 22, no. 1, pp. 51-68, 2015.

[10] M. R. Lovell, M. M. Khonsari, and R. D. Marangoni, "Low-speed friction torque on balls undergoing rolling motion," Tribology Transactions, vol. 36, no. 2, pp. 290-296, 1993.

[11] L. Houpert, "Ball bearing and tapered roller bearing torque: Analytical, numerical and experimental results," Tribology Transactions, vol. 45, no. 3, pp. 345-353, 2002.

[12] V. Wikström and E. Höglund, "Starting and steady-state friction torque of grease-lubricated rolling element bearings at low temperatures-part II: Correlation with less-complex test methods@)," Tribology Transactions, vol. 39, no. 3, pp. 684-690, 1996.

[13] T. Cousseau, B. Graça, A. Campos, and J. Seabra, "Experimental measuring procedure for the friction torque in rolling bearings," Lubrication Science, vol. 22, no. 4, pp. 133-147, 2010.

[14] C. M. C. G. Fernandes, P. M. P. Amaro, R. C. Martins, and J. H. O. Seabra, "Torque loss in cylindrical roller thrust bearings lubricated with wind turbine gear oils at constant temperature," Tribology International, vol. 67, pp. 72-80, 2013.

[15] C. Wang, R. Ning, J. Liu, and T. Zhao, "Dynamic simulation and disturbance torque analyzing of motional cable harness based on Kirchhoff rod model," Chinese Journal of Mechanical Engineering, vol. 25, no. 2, pp. 346-354, 2012.

[16] C. S. Wang, J. H. Liu, R. X. Ning et al., "Experimental validation method of elastic thin rod model for simulating the motional cable harness," Journal of Beijing Institute of Technology, vol. 21, no. 2, pp. 193-197, 2012 (Chinese).
[17] R. Cubalchini, "Technology and design needs for precision pointing systems," in Proceedings of the 34th Conference on Design \& Control, pp. 13-15, New Orleans, LA, USA, December 1995.

[18] L. Stockum, J. Profeta, and L. Ballou, "Precision stabilization system design to reduce the effects of friction," in Proceedings of the SPIE conference on Acquisition, Tracking, and Pointing II, pp. 159-167, Los Angeles, CA,USA, January 1988.

[19] C. McKerley, "A model for a two degree of freedom coupled seeker with mass imbalance," in Proceedings of the IEEEon Bringing Together Education, Science and Technology, pp. 84-87, Tampa, FL, USA, April 1996.

[20] M. M. Abdo, A. R. Vali, A. R. Toloei, and M. R. Arvan, "Improving two axes gimbal seeker performance using cascade control approach," Proceedings of the Institution of Mechanical Engineers, Part G: Journal of Aerospace Engineering, vol. 229, no. 1, pp. 38-55, 2015.

[21] M. Abdo, A. R. Vali, A. Toloei, and M. R. Arvan, "Research on the cross-coupling of a two axes gimbal system with dynamic unbalance," International Journal of Advanced Robotic Systems, vol. 10, no. 357, pp. 1-13, 2013.

[22] M. M. Abdo, A. R. Vali, A. R. Toloei, and M. R. Arvan, "Stabilization loop of a two axes gimbal system using self-tuning PID type fuzzy controller," ISA Transactions, vol. 53, no. 2, pp. 591-602, 2014.

[23] B. Armstrong-Hélouvry, P. Dupont, and C. C. de Wit, "A survey of models, analysis tools and compensation methods for the control of machines with friction," Automatica, vol. 30, no. 7, pp. 1083-1138, 1994.

[24] J. Yang, A. Plummer, and Y. Xue, "Dynamic friction modelling without drift and its application in the simulation of a valve controlled hydraulic cylinder system," Journal of Advanced Mechanical Design, Systems, and Manufacturing, vol. 8, no. 6, pp. 1-6, 2014.

[25] J. O. Östensen, H. Åström, and E. Höglund, "Analysis of a Grease-Lubricated Roller Bearing under Arctic Conditions," Journal of Engineering Tribology, vol. 209, no. 3, pp. 213-220, 2006.

[26] F. J. Du, J. Zhang, and H. K. Wen, "Analysis, testing and control of telescope's high-precision drive system in low-temperature environment," in Proceedings of the SPIE conference on Advances in Optical and Mechanical Technologies for Telescopes and Instrumentation, vol. 91513B, Quebec, Canada, June 2014.

[27] T. A. Harris, Rolling Bearing Analysis, John Wiley and Sons, Hoboken, NJ, USA, 3rd edition, 2001.

[28] J. X. Xu, Study on the Friction Torque Characteristics of Angular Contact Ball Bearings, Lanzhou University of Technology, 2014.

[29] T. C. Lim and R. Singh, "Vibration transmission through rolling element bearings, part I: bearing stiffness formulation," Journal of Sound and Vibration, vol. 139, no. 2, pp. 179-199, 1990. 


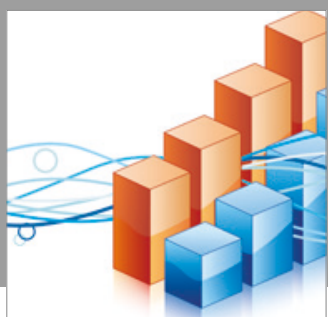

Advances in

Operations Research

vatersals

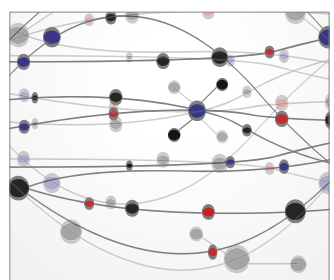

\section{The Scientific} World Journal
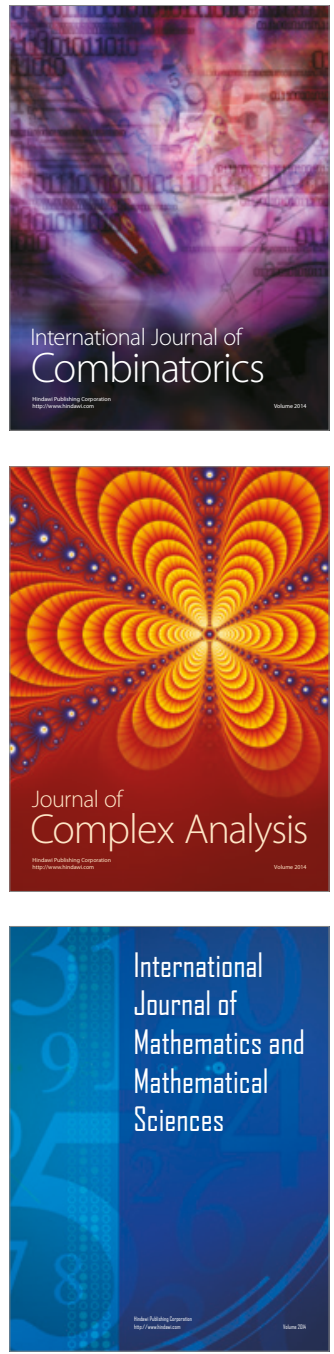
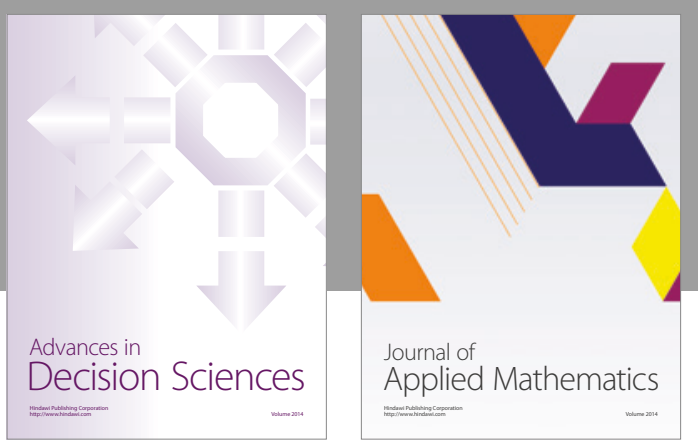

Algebra

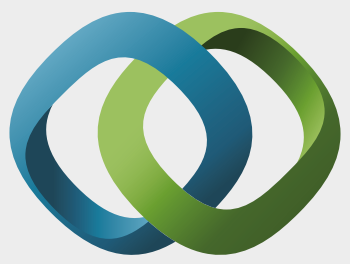

\section{Hindawi}

Submit your manuscripts at

https://www.hindawi.com
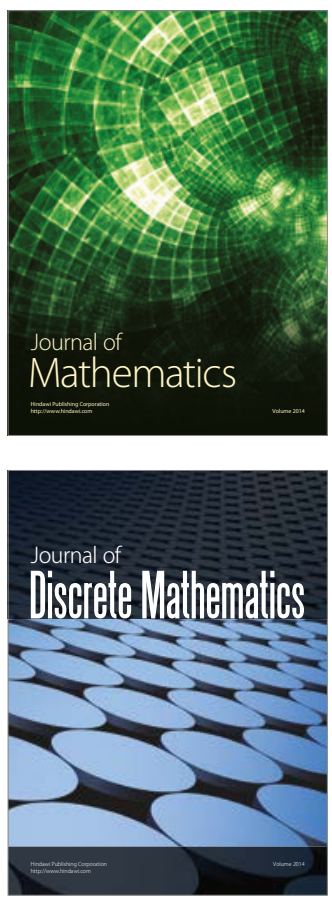

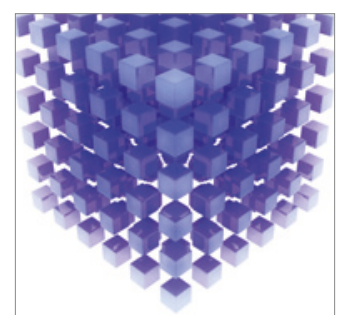

Mathematical Problems in Engineering
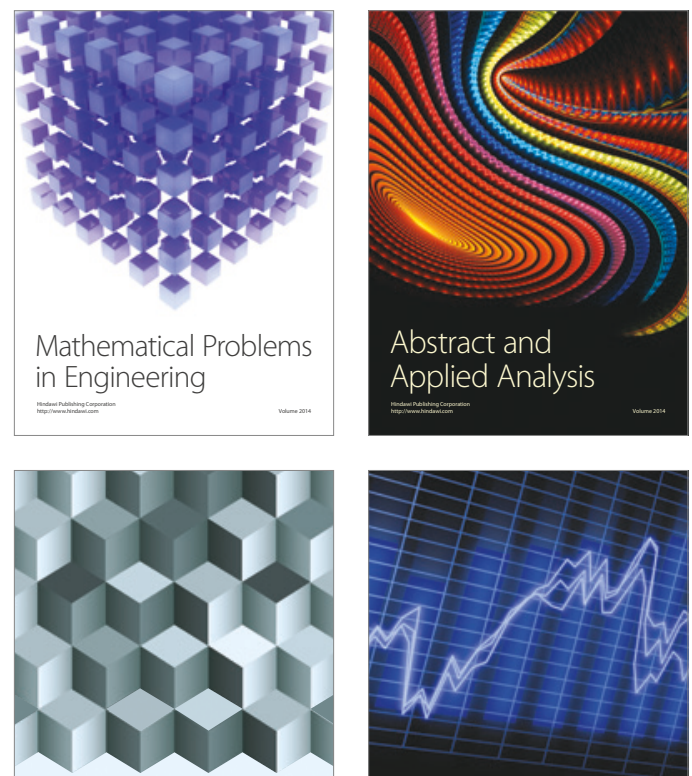

Journal of

Function Spaces

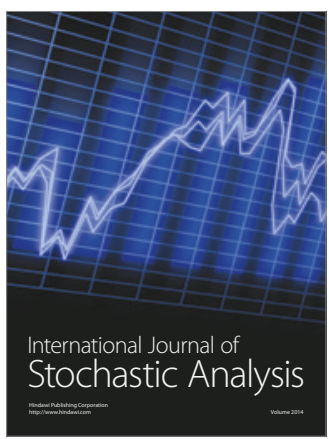

Probability and Statistics
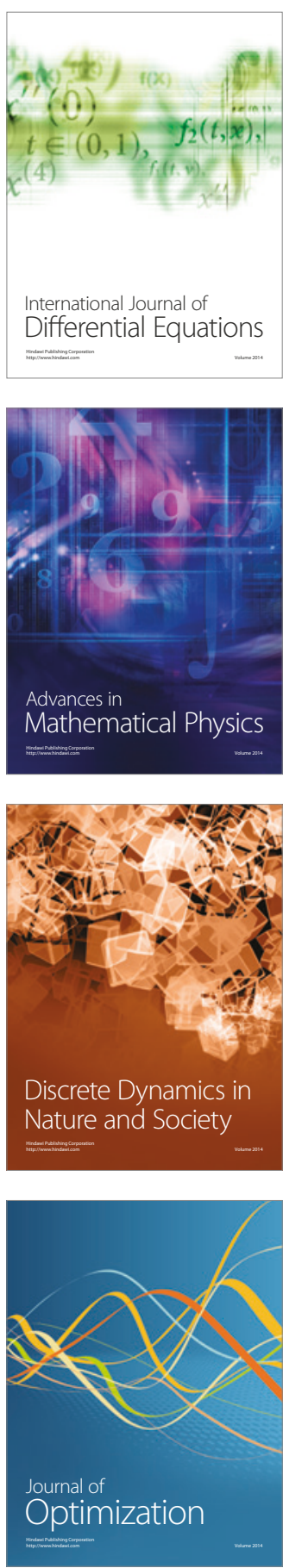